\title{
Immobilization of DNA on Quartz Crystal Microbalance Sensor Modified with Self-Assembled Monolayer of Thiol Derivative
}

\author{
Hirobumi Shibata, 2* , Junpei Nemoto ${ }^{1}$, Shota Shiba ${ }^{1}$, Yusuke Yamane ${ }^{1}$, Gota Kawai ${ }^{3}$, \\ and Kazuaki Hashimoto ${ }^{1}$ \\ ${ }_{1}^{1}$ Faculty of Engineering, Chiba Institute of Technology, 2-17-1 Tsudanuma, Narashino, Chiba 275-0016, JAPAN \\ ${ }^{2}$ Photocatalysis International Research Center, Tokyo University of Science, 2641 Yamazaki, Noda, Chiba 278-8510, JAPAN \\ ${ }^{3}$ Faculty of Advanced Engineering, Chiba Institute of Technology, 2-17-1 Tsudanuma, Narashino, Chiba 275-0016, JAPAN
}

\begin{abstract}
In this study, we investigated the direct detection of DNA, without pretreatment, using a quartz crystal microbalance (QCM) sensor. This sensor is modified by a self-assembled monolayer of a thiol derivative that has an amino group as the terminal functional group. Contact angle values and the attenuated total reflectance Fourier transform infrared (ATR/FT-IR) spectra of the QCM sensors after immersion into an ethanol solution of thiol derivatives clearly showed that self-assembled monolayers of the derivatives were formed on the QCM sensors. Although QCM measurements using unmodified and carboxylic group-modified sensors could not detect DNA-Na salt, the sensor modified with amino groups could detect the DNA. This system can be used for the analysis of the interaction between DNA and DNAbinding proteins.
\end{abstract}

Key words: quartz crystal microbalance, self-assembled monolayer, DNA, thiol derivative

\section{Introduction}

The development of analytical methods for measuring the interaction between biomolecules is very important to the understanding of biological activities. Many tools for elucidating biomolecular interactions have been investigated. Nuclear magnetic resonance (NMR) provides information on molecular structures, conformational changes, and intermolecular interactions ${ }^{1-5)}$. Since different molecules have different bonds and conjugation patterns, UV-vis spectroscopy holds promise for monitoring biomolecular interactions ${ }^{6-10)}$. Fluorescence resonance energy transfer (FRET) applied to biosensors enables the detection of interactions with high sensitivity and accommodates a wide range of donor and acceptor molecules ${ }^{11-15)}$. Surface plasmon resonance (SPR) is an optical-sensing method that measures changes in the refraction of a thin metal film surface upon binding with a given molecule ${ }^{16-18)}$.

Recent DNA technology is very advanced ${ }^{19-21)}$, for example, DNA damage response(DDR)and/or increasing replication stress result in genomic instability which is a key property of cancer ${ }^{22}$. Therefore, the detection of DNA is one of the most important research projects. Quartz crystal microbalance (QCM) measurement is one of the most powerful tools for providing qualitative and quantitative information about biomolecular interactions by the detection of mass change ${ }^{23,24)}$. Although the adsorption of DNA on QCM sensors is an important technique for the elucidation of replication and transcription processes, DNA is not directly adsorbed by Au on the QCM sensors. One of the most popular techniques to overcome this problem is using an avidin-bound QCM surface and biotinylated $\mathrm{DNA}^{25,26)}$. However, this technique requires a complicated process to synthesize biotinylated DNA. Thus, the immobilization of DNA on QCM sensors without pretreatment is one of the most important challenges for the detection and investigation of the relationship between DNA and biomolecules using QCM measurements.

Molecules having thiol groups form self-assembled monolayers (SAMs) with high density and degree of orientation on Au substrates ${ }^{27,28)}$. SAMs can be formed by dipping the substrates into a solution containing thiol derivatives. In addition, many thiol derivatives have various terminal functional groups. These derivatives promise to enable facile surface modification of $\mathrm{Au}$ with various terminal

*Correspondence to: Hirobumi Shibata, Faculty of Engineering, Chiba Institute of Technology, 2-17-1 Tsudanuma, Narashino, Chiba 275-0016, JAPAN

E-mail: hirobumi.shibata@it-chiba.ac.jp

Accepted January 10, 2020 (received for review December 6, 2019)

Journal of Oleo Science ISSN 1345-8957 print / ISSN 1347-3352 online

http://www.jstage.jst.go.jp/browse/jos/ http://mc.manusriptcentral.com/jjocs 


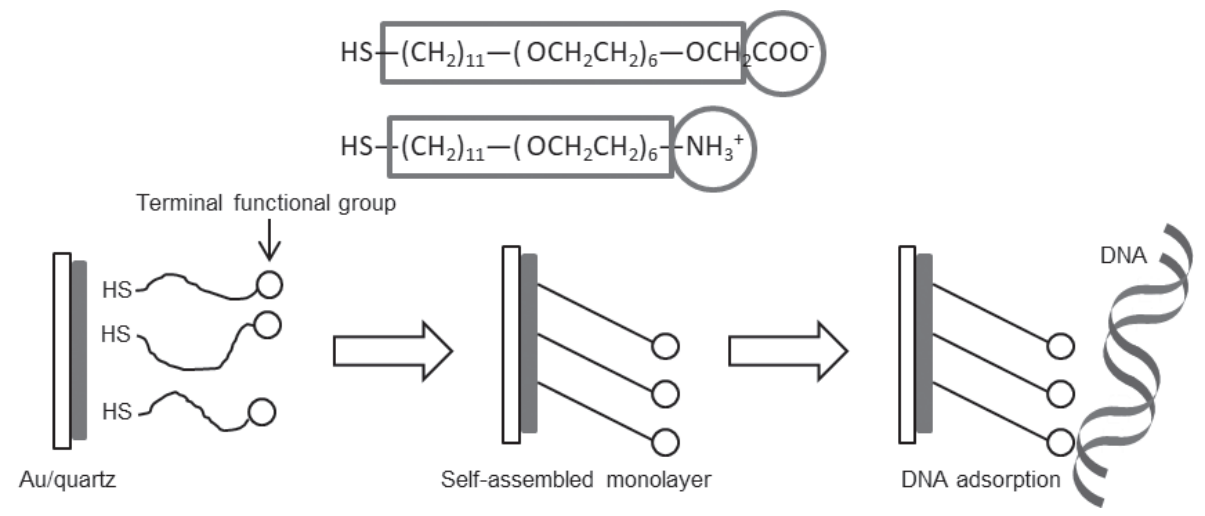

Fig. 1 Schematic illustration of DNA detection using sensor modified with thiol derivative.

functional groups. The modification of $\mathrm{Au}$ on the surface of the QCM sensors with thiol derivatives allows the adsorption and immobilization of biomolecules with facile pretreatment. This also indicates that DNA without pretreatment can be adsorbed on a QCM sensor due to the interaction between DNA and functional groups on the sensor. Furthermore, the mixing of thiol derivatives having various terminal functional groups can control the surface condition $^{29-31)}$ and promises the methodology for the detection of the strength of interaction between biomolecules and functional groups on sensors. Here, we demonstrate a novel detection technique for DNA, as shown in Fig. 1. In this study, we prepared QCM sensors modified with thiol derivatives and investigated the detection of DNA molecules without pretreatment based on their adsorption and immobilization on the QCM sensors.

\section{Experimental Procedures}

\subsection{Materials}

$\mathrm{HS}-\left(\mathrm{CH}_{2}\right)_{11}-\left(\mathrm{OCH}_{2} \mathrm{CH}_{2}\right)_{6}-\mathrm{OCH}_{2} \mathrm{COOH}$ and $\mathrm{HS}-$ $\left(\mathrm{CH}_{2}\right)_{11}-\left(\mathrm{OCH}_{2} \mathrm{CH}_{2}\right)_{6}-\mathrm{NH}_{2} \cdot \mathrm{HCl}$ (Altech Co. $)$ were used to modify the QCM sensors. These thiol derivatives have carboxylic and amino groups as terminal functional groups, respectively. Sodium chloride, sodium dihydrogen-phosphate, and disodium hydrogen-phosphate were purchased from Wako Pure Chemical Industries, Ltd. and used for the synthesis of phosphate buffered saline (PBS). DNA-Na salt originating from salmon soft roe (approximately 20,000 Da; Nissei Bio Co., Ltd.) was used for the investigation of DNA immobilization on the QCM sensors.

\subsection{Fabrication of PBS at $\mathrm{pH} 6.7$}

PBS at pH 6.7 was prepared as follows: A total of $14 \mathrm{~mL}$ of $100 \mathrm{mM}$ sodium dihydrogen-phosphate aqueous solution was mixed with $16 \mathrm{~mL}$ of $100 \mathrm{mM}$ disodium hydrogenphosphate aqueous solution. Then, $270 \mathrm{~mL}$ of distilled water was added to the solution. Finally, $8 \mathrm{~mL}$ of this mixture and $12 \mathrm{~mL}$ of distilled water were added to $20 \mathrm{~mL}$
Table 1 Contact angles for water on the sensors before and after surface modification with thiol derivatives.

\begin{tabular}{ccc}
\hline unmodified [ $^{\circ}$ ] & carboxylic group [ $\left.^{\circ}\right]^{\prime}$ & amino group [ ${ }^{\circ}$ ] \\
\hline $57( \pm 2)$ & $67( \pm 3)$ & $83( \pm 3)$ \\
\hline
\end{tabular}

of $100 \mathrm{mM} \mathrm{NaCl}$ aqueous solution and de-aerated.

\subsection{Preparation of QCM sensors modified with thiol de- rivatives}

QCM sensors $\left(13 \mathrm{~mm}^{2}\right)$ were immersed in $5 \mu \mathrm{M}$ ethanol solutions with $0.35 \mathrm{~mL}$ of the thiol derivatives at room temperature for $24 \mathrm{~h}$. Afterwards, the sensors were washed with ethanol. The obtained sensors were characterized by contact angle measurements using $2 \mu \mathrm{L}$ water droplets, and by Fourier transform infrared spectroscopy (FT-IR; JASCO, FT/IR-4200) using the attenuated total reflectance (ATR) method.

\subsection{QCM measurements using the sensors modified with thiol derivatives}

The immobilization of DNA using the NAPiCOS system with a QCM twin sensor (Nihon Dempa Kogyo Co., Ltd.) was investigated as follows: QCM sensors modified with thiol derivatives were placed in PBS. The flow rate of the PBS was fixed at $0.05 \mathrm{~mL} / \mathrm{min}$. After the stabilization of the QCM frequency, $5.0 \times 10^{-5} \mathrm{~g} / \mathrm{mL}$ DNA-Na salt PBS solution was injected and the in-situ QCM frequency shifts were monitored as a function of time.

\section{Results}

\subsection{Preparation of QCM sensors modified with thiol de- rivatives}

First, we carried out contact angle measurements of the QCM sensors after immersion in ethanol solutions with the thiol derivatives. Table 1 shows the contact angles for 


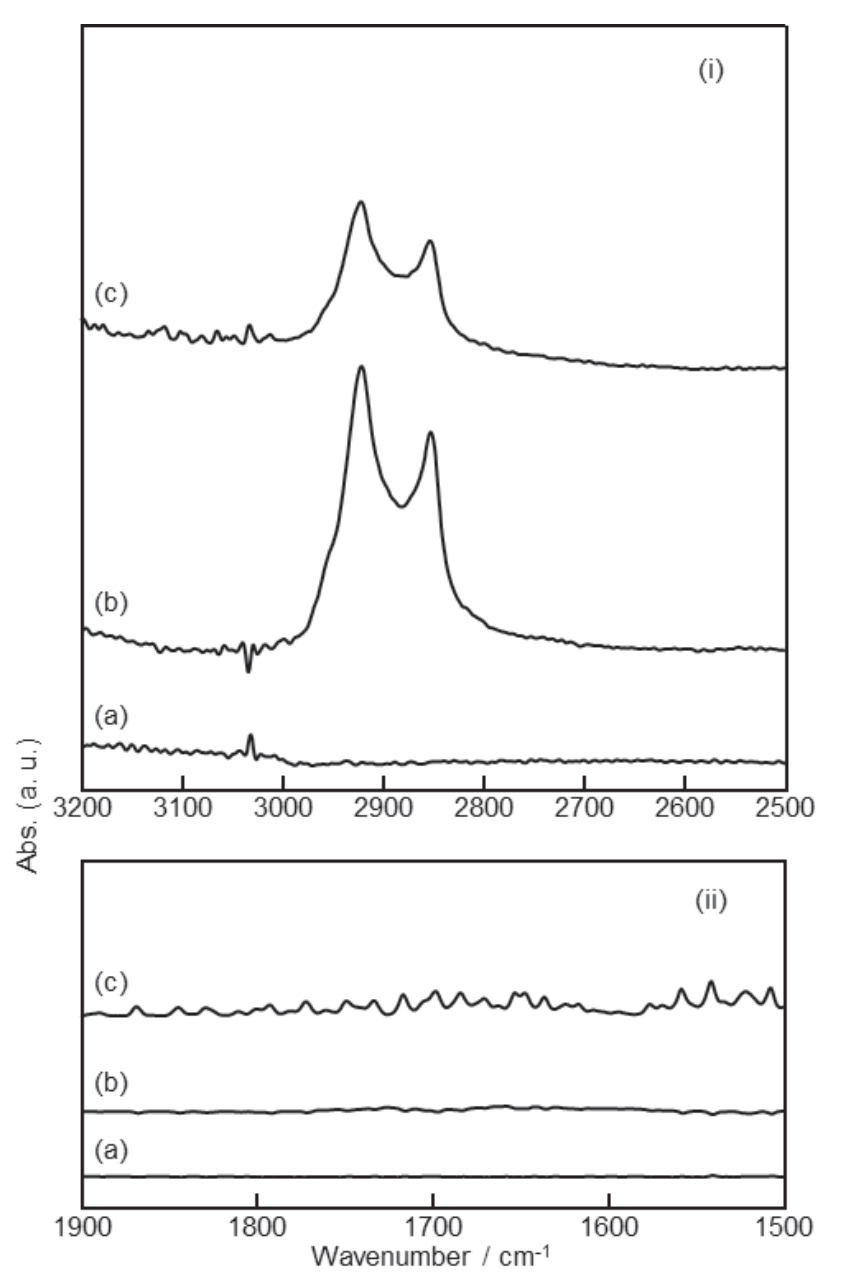

Fig. 2 ATR/FT-IR spectra of (a) unmodified and (b, c) modified sensors. (b) and (c) show the ATR/FT-IR spectra of the sensors modified with thiol derivatives having carboxyl and amino groups, respectively. Panels (i) and (ii) are for the regions $3200-2500$ and $1900-1500 \mathrm{~cm}^{-1}$, respectively.

water on unmodified QCM sensors and on sensors modified with thiol derivatives. The contact angle values on the modified sensors were higher than that of the unmodified sensor. These results suggested that the QCM sensors were modified with SAMs of thiol derivatives.

Moreover, we investigated the formation of SAMs of thiol derivatives on QCM sensors using ATR/FT-IR spectroscopy measurements. Figure 2 (i) shows the ATR/FT-IR spectra of unmodified and thiol derivative-modified QCM sensors. Although no peaks assigned to thiol derivatives were detected in the ATR/FT-IR spectrum of the unmodified QCM sensor (Fig. 2(i) (a)), peaks due to $\mathrm{C}-\mathrm{H}$ stretching vibrations of thiol derivatives appeared at approximately 2850 and $2920 \mathrm{~cm}^{-1}$ in ATR/FT-IR spectra of the modified sensors with thiol derivatives having carboxylic (Fig. 2(i) (b) ) and amino (Fig. 2(i) (c) ) groups, respectively. These results demonstrated that the QCM sensors were modified

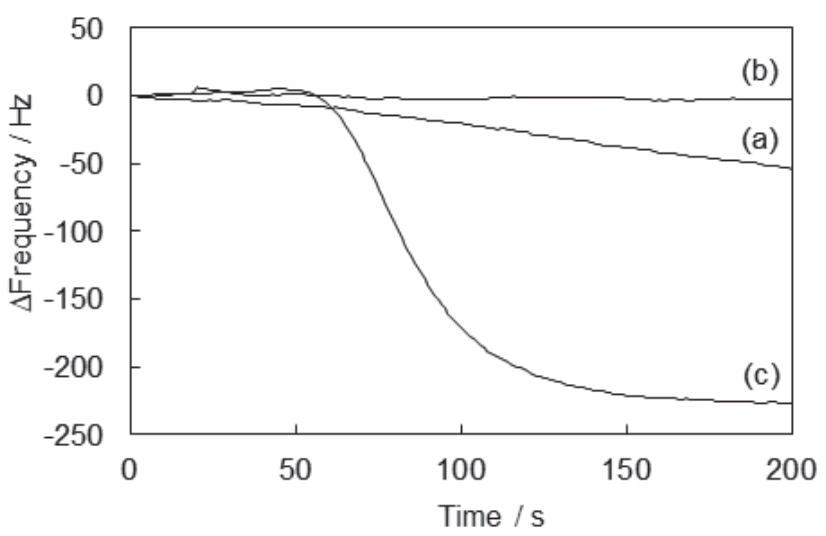

Fig. 3 Frequency changes on (a) unmodified and (b, c) modified sensors. (b) and (c) show the results for sensors modified with carboxyl and amino groups, respectively.

with SAMs of thiol derivatives having carboxylic and amino groups.

\subsection{QCM measurements using the sensors modified with thiol derivatives}

We carried out the QCM measurements using the modified sensors to investigate DNA detection based on its adsorption. Figure 3 shows the frequency changes for DNA adsorption onto the unmodified and modified QCM sensors. The time of DNA injection is indicated as $0 \mathrm{~min}$. When we used an unmodified sensor, a slight frequency decrease was observed (Fig. 3(a)). Here, DNA does not possess functional groups that are able to interact with $\mathrm{Au}$. Therefore, this decrease is considered to be based on the adsorption of impurities. In the case of the carboxylic group-modified sensor, a frequency change was not observed, and the value stayed constant (Fig. 3(b)). This means that DNA was not adsorbed on the sensor modified with thiol derivatives having carboxylic groups. These results indicate that DNA was not adsorbed and immobilized on these sensors. Figure 3(c) shows the result of QCM measurements using the sensor modified with amino groups. A large decrease in frequency of approximately $250 \mathrm{~Hz}$ was observed after approximately $60 \mathrm{~s}$ from the time of DNA injection. After this decrease, the frequency became constant. The mass of adsorbent calculated by Sauerbrey's equation was $120 \mathrm{ng} /$ $\mathrm{cm}^{2}$. These results suggested that DNA was adsorbed and immobilized on the sensor modified with the thiol derivative having amino groups.

Afterwards, in order to identify the adsorbents on the sensors, we carried out ATR/FT-IR measurements of the sensors immersed in a $5.0 \times 10^{-5} \mathrm{~g} / \mathrm{mL}$ DNA-Na salt PBS solution for $10 \mathrm{~min}$. The spectra of the sensors are shown in Fig. 4. Although ATR/FT-IR spectra of the sensors before immersion in a DNA-Na salt solution (Fig. 2 (ii)) have no peak in the region between $1800-1700 \mathrm{~cm}^{-1}$, a broadened 


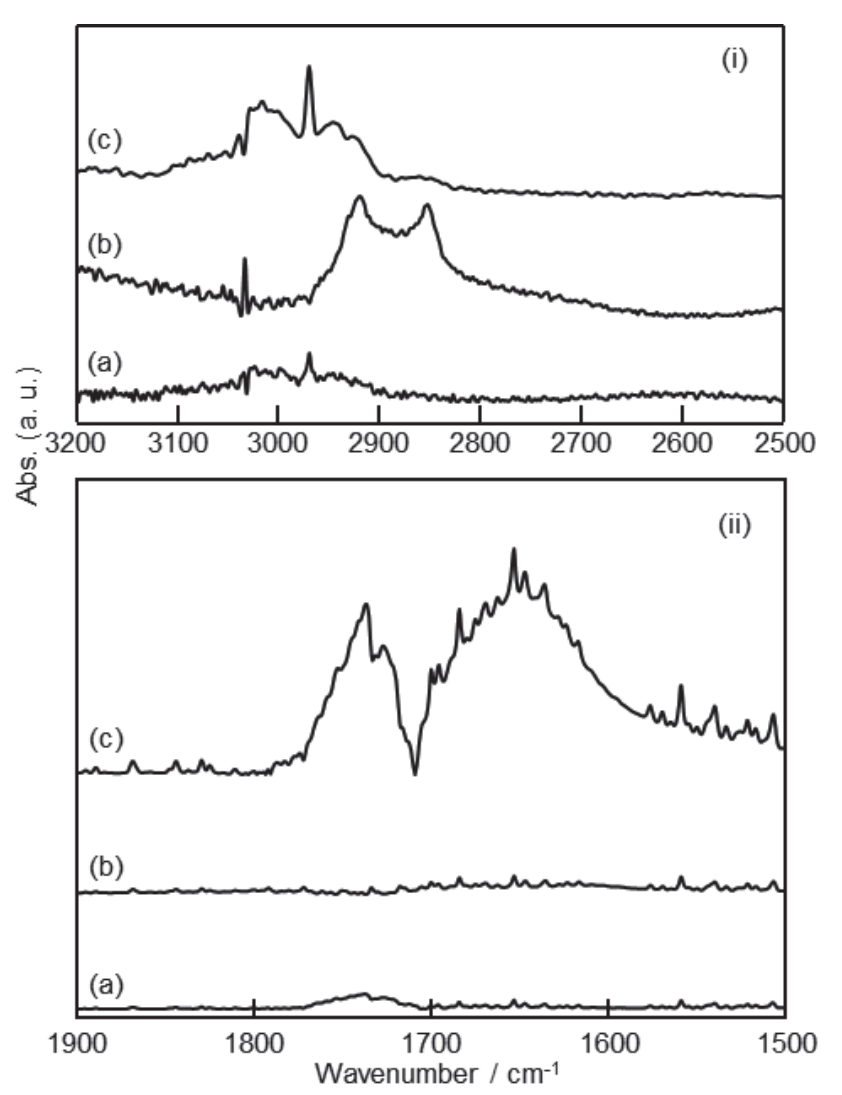

Fig. 4 ATR/FT-IR spectra of (a) unmodified and (b, c) modified sensors after immersion in DNA-Na salt PBS solution. (b) and (c) show the ATR/FT-IR spectra of sensors modified with thiol derivatives having carboxyl and amino groups, respectively. Panels (i) and (ii) are for the regions 3200-2500 and 1900-1500 $\mathrm{cm}^{-1}$, respectively.

peak was observed in this region in ATR/FT-IR spectra of unmodified and amino group-modified sensors (Fig. 4 (ii) $(\mathbf{a}, \mathbf{c}))$. This region has peaks assigned to carbonyl stretching vibrations, excluding amide bonds. This suggests that impurities, such as fatty acids, were adsorbed onto these sensors, and that the frequency decrease of the unmodified sensor was related with this adsorption. Next, we focused on the region $1700-1600 \mathrm{~cm}^{-1}$. The peak assigned to DNA appears in this region ${ }^{32,33)}$. Although the ATR/FT-IR spectra of unmodified and carboxylic group-modified sensors shown in Fig. 4(ii) (a) and (b) do not have this peak, the peak is present in the spectrum of the sensor modified with the thiol derivative having amino groups (Fig. 4 (ii) (c)). Subsequently, we focused on the ATR/FT-IR spectra in the region $3200-2500 \mathrm{~cm}^{-1}$. The peaks assigned to the thiol derivatives were detected in the ATR/FT-IR spectrum of the carboxylic-group modified sensor(Fig. 4 (i) (b)). A broadened peak was also observed at approximately $2850 \mathrm{~cm}^{-1}$ in the spectrum of the sensor modified with amino groups (Fig. 4(i) (c)). The same broadened peaks were observed in the region $3050-2900 \mathrm{~cm}^{-1}$ in ATR/ FT-IR spectra of unmodified and amino-group modified sensors. From the results mentioned so far, these broadened peaks are originated from impurities. Adsorption of impurities resulted in the broadening of the peak assigned to the thiol derivative having amino groups. These results clearly showed that the SAMs of thiol derivatives remained on QCM sensors after immersion in a DNA-Na salt solution. This means that DNA was adsorbed and immobilized on the sensors due to the interaction between DNA and the amino group of the thiol derivative. These results are in good agreement with the frequency changes shown in Fig. 3. The results of QCM and ATR/FT-IR measurements clearly reveal that by using a sensor modified with amino groups, we can detect DNA, such as biotinylation, without the need for pretreatment.

The above results can be explained by considering mainly the zeta potential of the terminal functional groups of the thiol derivatives and in one case the functional groups of DNA. DNA does not have any functional groups, such as the thiol group, that can form interactions with $\mathrm{Au}$. Therefore, unmodified sensors cannot detect DNA because they do not interact with DNA to enable adsorption. QCM measurements were carried out using PBS at $\mathrm{pH}$ 6.7. The sensor modified with carboxylic groups is negatively charged due to the dissociation of carboxylic groups. Electrostatic repulsive forces between carboxylic groups on the sensor and the phosphate groups of DNA led to their nondetection by QCM measurements. In contrast, amino groups on the sensor are positively charged at approximately $\mathrm{pH}=6^{34-37)}$. The electrostatic attractive force between the amino groups and the phosphate groups in DNA results in the adsorption and immobilization of DNA on the sensor.

\section{Conclusions}

This study demonstrated a facile technique for DNA detection due to adsorption and immobilization of DNA using QCM sensors modified with SAMs of thiol derivatives. We succeeded in the preparation of QCM sensors modified with SAMs of thiol derivatives having carboxylic and amino groups. The amino group-modified sensor could detect DNA-Na salt without pretreatment. The adsorption and immobilization of DNA on the sensor took place due to the electrostatic attractive force between amino groups on the sensor and the DNA-Na salt. These results suggested that the choice of an appropriate terminal functional group on thiol derivatives can lead to the stable immobilization of various biomolecules on $\mathrm{Au}$. In addition, QCM measurements using sensors modified with SAMs of thiol derivatives can detect mass changes due to interactions between biomolecules and terminal functional groups of thiol deriv- 
atives without the need for complicated pretreatment. This method holds promise as an important technique for the facile detection of biomolecules and the investigation of biomolecular interactions.

\section{Acknowledgment}

This work was supported by Grants-in-Aid for Scientific Research on Innovative Areas (21200039) from the Ministry of Education, Culture, Sports, Science and Technology.

\section{References}

1) Bertini, I.; Turano, P.; Vila, A.J. Nuclear magnetic resonance of paramagnetic metalloproteins. Chem. Rev. 93, 2833-2932 (1993).

2) Wang, Q.; Zhuravleva, A.; Gierasch, L.M. Exploring weak, transient protein - protein interactions in crowded in vivo environments by in-cell nuclear magnetic resonance spectroscopy. Biochemistry 50, 92559236 (2010).

3) Otabe, T.; Nagano, K.; Kawai, G.; Murata, A.; Nakatani, K. Inhibition of pre-miRNA-136 processing by Dicer with small molecule BzDANP suggested the formation of ternary complex of pre-miR-136-BzDANP-Dicer. Bioorg. Med. Chem. 27, 2140-2148(2019).

4) Amano, R.; Aoki, K.; Miyakawa, S.; Nakamura,Y.; Kozu, T.; Kawai, G.; Sakamoto, T. NMR monitoring of the SELEX process to confirm enrichment of structured RNA. Sci. Rep. 7, 283 (2017).

5) Otsu, M.; Kajikawa, M.; Okada, N.; Kawai, G. Solution structure of a reverse transcriptase recognition site of a LINE RNA from zebrafish. J. Biochem. 162, 279-285 (2017).

6) Hu, Y.-J.; Liu, Y.; Zhao, R.-M.; Dong, J.-X.; Qu, S.-S. Spectroscopic studies on the interaction between methylene blue and bovine serum albumin. J. Photochem. Photobiol. A-Chem. 179, 324-329 (2006).

7) Hu, Y.-J.; Liu, Y.; Wang, J.-B.; Xiao, X.-H.; Qu, S.-S. Study of the interaction between monoammonium glycyrrhizinate and bovine serum albumin. J. Pharm. Biomed. Anal. 36, 915-919(2004).

8) Wang, Y.-Q.; Zhang, H.-M.; Zhang, G.-C.; Tao, W.-H.; Tang, S.-H. Interaction of the flavonoid hesperidin with bovine serum albumin: A fluorescence quenching study. J. Lumin. 126, 211-218(2007).

9) Li, N.; Ma, Y.; Yang, C.; Guo, L.; Yang, X. Interaction of anticancer drug mitoxantrone with DNA analyzed by electrochemical and spectroscopic methods. Biophys. Chem. 116, 199-205 (2005).

10) Bi, S.; Zhang, H.; Qiao, C.; Sun, Y.; Liu, C. Studies of interaction of emodin and DNA in the presence of ethid- ium bromide by spectroscopic method. Spectrochim. Acta A-Mol. Biomol. Spectrosc. 69, 123-129 (2008).

11) Wang, Y.; Bao, L.; Liu, Z.; Pang, D.-W. Aptamer biosensor based on fluorescence resonance energy transfer from upconverting phosphors to carbon nanoparticles for thrombin detection in human plasma. Anal. Chem. 83, 8130-8137 (2011).

12) Wang, Y.; Shen, P.; Li, C.; Wang, Y.; Liu, Z. Upconversion fluorescence resonance energy transfer based biosensor for ultrasensitive detection of matrix metalloproteinase-2 in blood. Anal. Chem. 84, 1466-1473 (2012).

13) Wu, Y.-M.; Cen, Y.; Huang, L.-J.; Yu, R.-Q.; Chu, X. Upconversion fluorescence resonance energy transfer biosensor for sensitive detection of human immunodeficiency virus antibodies in human serum. Chem. Commun. 50, 4759-4762(2014).

14) Massey, M.; Algar, W.R.; Krull, U.J. Fluorescence resonance energy transfer (FRET) for DNA biosensors: FRET pairs and Förster distances for various dyeDNA conjugates. Anal. Chim. Acta 568, 181-189 (2006).

15) Xu, L.; Zhu, Y.; Ma, W.; Kuang, H.; Liu, L.; Wang, L.; $\mathrm{Xu}, \mathrm{C}$. Sensitive and specific DNA detection based on nicking endonuclease-assisted fluorescence resonance energy transfer amplification. J. Phys. Chem. C 115, 16315-16321 (2011).

16) Rich, R.L.; Myszka, D.G. Advances in surface plasmon resonance biosensor analysis. Curr. Opin. Biotechnol. 11, 54-61 (2000).

17) McDonnell, J.M. Surface plasmon resonance: towards an understanding of the mechanisms of biological molecular recognition. Curr. Opin. Chem. Biol. 5, 572577 (2001).

18) Law, W.-C.; Yong, K.-T.; Baev, A.; Prasad, P.N. Sensitivity improved surface plasmon resonance biosensor for cancer biomarker detection based on plasmonic enhancement. ACS Nano 5, 4858-4864 (2011).

19) Asanuma, H.; Murayama, K.; Kamiya, Y.; Kashida, H. The DNA duplex as an aqueous one-dimensional soft crystal scaffold for photochemistry. Bull. Chem. Soc. Jpn. 91, 1739-1748 (2018).

20) Kodera, M.; Kadoya, Y.; Aso, K.; Fukui, K.; Nomura, A.; Hitomi, Y.; Kitagishi, H. Acceleration of hydrolytic DNA cleavage by dicopper (II) complexes with $p$-cresol-derived dinucleating ligands at slightly acidic $\mathrm{pH}$ and mechanistic insights. Bull. Chem. Soc. Jpn. 92, 739-747 (2019).

21) Verschueren, D.V.; Pud, S.; Shi, X.; Angelis, L.D.; Kuipers, L.; Dekker, C. Label-free optical detection of translocations through plasmonic nanopores. ACS Nano 13, 61-70(2019).

22) Pilie, P.G.; Tang, C.; Mills, G.B.; Yap, T.A. State-of-theart strategies for targeting the DNA damage response 
in cancer. Nat. Rev. Clin. Oncol. 16, 81-104(2019).

23) Furusawa, H.; Ichimura, Y.; Harada, S.; Uematsu, M.; Xue, S.; Nagamine, K.; Tokito, S. Electric charge detection of sparse organic acid molecules using an organic field-effect transistor (OFET) -based sensor. Bull. Chem. Soc. Jpn. 91, 1020-1025 (2018).

24) Zhang, D.; Wang, D.; Li, P.; Zhou, X.; Zong, X.; Dong, G. Facile fabrication of high-performance QCM humidity sensor based on layer-by-layer self-assembled polyaniline/graphene oxide nanocomposite film. Sens. Actuators B: Chem. 255, 1869-1877 (2018).

25) Okahata, Y.; Niikura, K.; Sugiura, Y.; Sawada, M.; Morii, T. Kinetic studies of sequence-specific binding of GCN4-bZIP peptides to DNA strands immobilized on a 27-MHz quartz-crystal microbalance. Biochemistry 37, 5666-5672 (1998).

26) Niikura, K.; Matsuno, H.; Okahata, Y. Binding behavior of lysine-containing helical peptides to DNA duplexes immobilized on a $27 \mathrm{MHz}$ quartz-crystal microbalance. Chem.-Eur. J. 5, 1609-1616. (1999).

27) Wink, T.; Van Zuilen, S.J.; Bult, A.; Van Bennekom, W.P. Self-assembled monolayers for biosensors. Analyst 122, 43R-50R (1997).

28) Schwartz, D.K. Mechanisms and kinetics of self-assembled monolayer formation. Annu. Rev. Phys. Chem. 52, 107-137 (2001).

29) Mendes, P.M. Stimuli-responsive surfaces for bio-applications. Chem. Soc. Rev. 37, 2512-2529 (2008).

30) Chechik, V.; Crooks, R.M.; Stirling, C.J.M. Reactions and reactivity in self-assembled monolayes. $A d v . M a-$ ter. 12, 1161-1171 (2000).

31) Ulman, A. Wetting studies of molecularly engineered surfaces. Thin Solid Films 273, 48-53(1996).

32) Mao, Y.; Daniel, L.N.; Whittaker, N.; Saffiotti, U. DNA binding to crystalline silica characterized by Fouriertransform infrared spectroscopy. Environ. Health Perspect. 102, 165-171(1994).

33) Alex, S.; Dupuis, P. FT-IR and Raman investigation of cadmium binding by DNA. Inorg. Chim. Acta 157, 271-281 (1989).

34) Shyue, J.-J.; Guire, M.R.D. Acid-base properties and Zeta potentials of self-assembled monolayers obtained via in situ transformations. Langmuir 20, 8693-8698 (2004).

35) Shibata, H.; Sakai, T.; Sato, M.; Watanabe, S.; Matsumoto, M. Patterning of silica particles using self-assembly on two-dimensional patterns fabricated from phase-separated Langmuir-Blodgett films. Trans. $M a-$ ter. Res. Soc. Jpn. 35, 339-342 (2010).

36) Shibata, H.; Shinozaki, R.; Ogura, T.; Sakai, H.; Abe, M.; Kawai, G.; Hashimoto, K. Fabrication and BSA adsorption/desorption properties of Titania/Silica composite films modified with silane coupling agents. J. Oleo Sci. 63, 1077-1083 (2014).

37) Shibata, H. Fabrication and functionalization of inorganic materials using amphiphilic molecules. J. Oleo Sci. 66, 103-111(2017). 